\title{
Giant Magnetic Field Enhancement in Hybridized MIM Structures
}

\author{
Salma Alrasheed, ${ }^{1, *}$ Enzo Di Fabrizio ${ }^{1, *}$
}

${ }^{1}$ King Abdullah University of Science and Technology, PSE and BESE Divisions, Thuwal, 23955- 6900, Kingdom of Saudi Arabia

\begin{abstract}
We propose numerically an approach to narrow the plasmon linewidth and enhance the magnetic near field intensity at a magnetic hot spot in a hybridized metal-insulatormetal (MIM) structure. First we insert in part of the dielectric layer of the MIM, at its center, another dielectric material of a high refractive index (HRI). This results in an increase in the magnetic near field enhancement of the magnetic plasmon (MP) resonance by $82 \%$ compared with the MIM without the HRI material. We then couple this enhanced MP resonance to a propagating surface plasmon polariton (SPP) to achieve a further enhancement of $438 \%$. The strong coupling between the MP and the SPP is demonstrated by the large anti-crossing in the reflection spectra. The resulting maximum magnetic field enhancement at the gap is $\sim\left|H / H_{i}\right|^{2}=3555$.
\end{abstract}

Index Terms-Metamaterials, Plasmons, Magnetic field enhancement, Magnetic plasmons, Optical coupling.

\section{INTRODUCTION}

$\mathrm{A}_{\text {interactions are mainly through the electrical }}$ component of light, while the magnetic component effect is extremely weak [1]. However, it has been shown that artificial magnetism can be generated at optical and NIR frequencies through utilizing surface plasmon resonances (SPRs) in specially designed metamaterials. The incoming magnetic field of the wave can excite SPRs that can produce a current loop, which gives rise to an effective magnetic dipole moment that opposes the incident magnetic field $[2,3]$. Such magnetic plasmon resonance is different from LC resonances arising in perfectly conducting split-ring resonators (SRRs) at microwave and mid-IR frequencies, and in which the skin depth is much smaller than the structure size [2,3]. In such SRRs, the strong magnetic response is achieved at a particular ratio of the radiation wavelength to the structure size. However in magnetic plasmon resonances in subwavelength structures, the plasmonic effect, due to the size being comparable to the skin depth, plays the dominant role. The resonance ${ }^{1}$ frequency is determined by the electromagnetic properties of the metal and the structure geometry rather than the wavelength to size ratio. As a result, the magnetic plasmon resonance appears in the optical-NIR range of the spectrum $[2,3]$. Several structures have been proposed in the literature to demonstrate magnetic hot spots including nanorings [4], MIM nanosandwiches [5-7] and nanoparticle clusters [8,9]. The MIM structure (nanosandwich) consists of two metallic strips separated by a dielectric layer. Such structure supports an anti-symmetric mode where the electric field in the two metallic strips oscillates out of phase producing antiparallel currents which combine with the displacement currents in the dielectric gap to form a current loop that produces a magnetic dipole moment in a direction that is opposite to the incident magnetic field [7,10]. This results in a highly enhanced and localized magnetic field in the dielectric layer known as a magnetic hot spot with potential applications in sensors, light matter interaction at weak magnetic fields and negative refractive index materials $[3,11]$. One of the main problems in plasmonics is the high radiative damping resulting in a large plasmon linewidth which limits the near field enhancement $[12,13,14]$. Various methods have been used to reduce the radiative damping through coupling the LSP resonance to a narrow resonance mode. Some examples for enhancing the magnetic plasmon resonance include farfield diffractive coupling $[10,15]$, coupling to Bloch surface waves [16], coupling to SPPs [17] and coupling with a photonic microcavity $[18,19]$.

Here we propose a numerical approach to narrow the plasmon linewidth and enhance the magnetic near field intensity at a magnetic hot spot in a gold-insulator-gold (MIM) nanostructure. We first introduce in part of the dielectric layer of a single MIM on a glass substrate, at its

Copyright (c) 2017 IEEE. Personal use of this material is permitted. However, permission to use this material for any other purposes must be obtained from the IEEE by sending a request to pubs-permissions@ieee.org. 
center, another dielectric material with a HRI of 3. This results in an increase of the magnetic near field enhancement of the magnetic plasmon (MP) resonance by $35 \%$ compared to the same single MIM structure on a glass substrate, but with the only use of a dielectric spacer layer of an index of refraction of 1.45. For brevity, we will refer to the MIM with the added HRI as the hybridized MIM (HMIM). We then couple this enhanced MP resonance to a propagating surface plasmon polariton (SPP) to achieve a further enhancement of $438 \%$. The strong coupling between the MP and the SPP is demonstrated by the large anti-crossing in the reflection spectra with the generation of two hybridized MP modes. The resulting maximum enhanced magnetic field at the gap is $\left|H / H_{i}\right|^{2}=3554.8$ with a total of $796 \%$ increase in enhancement compared to the MIM (without using both the HIR layer and coupling with a SPP). The computational method used is the finite-difference time-domain method (FDTD) using the commercial software Lumerical Solutions Inc. (Ver. 8.7.0). The SPPs are excited by grating coupling where the reciprocal vector of the grating provides the extra momentum to couple with the SPPs $[12,20]$. When placed in a periodic array on top of a gold film on a glass substrate, the collective resonance of the array (without coupling to the SPP) blue shifts from that of a single HMIM on a glass substrate from a wavelength of $894.9 \mathrm{~nm}$ to that of 854.5 $\mathrm{nm}$. The anticrossing is observed when the magnetic resonance of the collective HMIM periodic array on a gold film on a glass substrate matches that of the SPP at a period of $837 \mathrm{~nm}$. We tune the resonance wavelength of the $(1,0)$ SPP mode by changing the period of the array while keeping the size of the HMIM structures constant.

\section{RESULTS AND ANALYSIS}

The single HMIM structure on a glass $\left(\mathrm{SiO}_{2}\right)$ substrate of a thickness of $140 \mathrm{~nm}$ and an index of refraction of 1.45 is shown in the inset of Fig. 1. The HMIM consists of two gold strips separated by a dielectric layer composed of two types of dielectrics. Each strip has a width (w) of $120 \mathrm{~nm}$ and a length (l) of $80 \mathrm{~nm}$. The height $\left(\mathrm{h}_{1}\right)$ of each gold strip is 40 $\mathrm{nm}$ while the height $\left(\mathrm{h}_{2}\right)$ of the dielectric layer is $20 \mathrm{~nm}$. The dielectric layer is composed of two materials as shown in the inset of Fig. 1. At the center is a dielectric with a high refractive index of 3 and a width of $40 \mathrm{~nm}$, while on the sides is a dielectric with an index of refraction of 1.45 with also a width of $40 \mathrm{~nm}$ for each. Fig. 1 also shows the reflectance spectrum of this single HMIM structure. The peak at the wavelength of $689 \mathrm{~nm}$ corresponds to the excitation of the electric dipole (higher energy symmetric mode) when the currents in the strips are parallel, while the peak at the wavelength of $883 \mathrm{~nm}$ corresponds to the magnetic dipole moment (lower energy anti-symmetric mode) when the currents in the strips are anti-parallel.
For the simulation of this single structure, a TM plane wave total field scattered field source (TFST) illuminates the single MIM structure from above at normal incidence along the $z$-direction with a wavelength ranging from 600 to 1000 $\mathrm{nm}$. We use perfectly matched layer boundary conditions (PML) in all directions of the simulation region. The mesh accuracy is set to 3 which corresponds to a $\lambda / 14$ step size. A mesh override region of $1 \mathrm{~nm}$ is placed around the HMIM structure. To measure the reflectance in Fig. 1, a 2D frequency-domain power monitor, normal to the $\mathrm{z}$ direction (in the x-y plane), is placed at a distance of $275 \mathrm{~nm}$ above the structure. For the magnetic near field enhancement plots, a $2 \mathrm{D}$ frequency-domain profile monitor, that is normal to the $y$ direction (in the x-z plane), is placed at the center of the structure, where its boundaries are the same as that of the structure.

The complex refractive index of gold is taken from Johnson and Christy [21]. The incident plane wave has an electric field amplitude of $\left|E_{i}\right|=1 \mathrm{~V} / \mathrm{m}$ and a corresponding incident magnetic field amplitude of $\left|H_{i}\right|=2.654 \times 10^{-3} \mathrm{~A} / \mathrm{m}$.

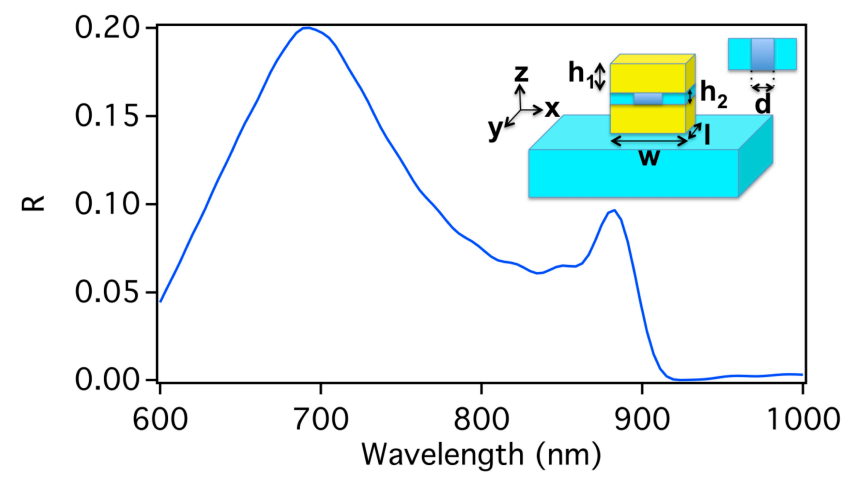

Fig. 1. Reflectance spectrum of the single HMIM structure (the amount of power reflected normalized to the source power). The peak at the wavelength of $689 \mathrm{~nm}$ corresponds to the excitation of the electric dipole moment, while the peak at the wavelength of $883 \mathrm{~nm}$ corresponds to the magnetic dipole moment. In the inset is a schematic representation of the single MIM structure on a glass substrate. Each strip has a width (w) of $120 \mathrm{~nm}$ and a length (l) of $80 \mathrm{~nm}$. The height $\left(\mathrm{h}_{1}\right)$ of each gold strip is $40 \mathrm{~nm}$ and the height $\left(\mathrm{h}_{2}\right)$ of the dielectric layer is $20 \mathrm{~nm}$. The dielectric layer is composed of two materials as shown in the inset. At the center is a dielectric with a high refractive index of 3 and a width (d) of $40 \mathrm{~nm}$, while on each side is a dielectric with an index of refraction of 1.45 and a width of $40 \mathrm{~nm}$ each.

Fig. 2 (a) shows the magnetic near field $|H|$ at the magnetic resonance at a wavelength of $850.5 \mathrm{~nm}$, for a single MIM on a glass substrate without using the center HRI material (only the dielectric with 1.45 index). Fig. (b) shows the near electric field $|E|$ at the same magnetic resonance of (a) and the corresponding electric field vectors. An enhancement factor of $\left.\left|H_{H}\right|_{i}\right|^{2}=446.6$ at the hot spot is observed. Fig. 2 (c) shows the magnetic near field $|H|$ at the magnetic resonance at a wavelength of 895 for a single HMIM on a glass substrate where the width (d) of the center HRI material is $40 \mathrm{~nm}$. Fig. 2 (d) shows the corresponding 
near electric field $|E|$ of the resonance in (c) and the corresponding electric field vectors. The magnetic near field enhancement of the HMIM is $\left|H / H_{i}\right|^{2}=811.7$ which is $82 \%$ higher than in the case when no center HRI material is used. In addition, the electric field enhancement at the gaps is reduced from $\left|E / E_{i}\right|^{2}=2065.4$ to $\left|E / E_{i}\right|^{2}=1701.6$ when the HRI is introduced and the frequency is redshifted. Since the MIM structures in general can be considered as a resonant LC circuit with the metal strips and the gaps at the ends as inductors and capacitors respectively [2,11], it appears that inserting the HRI material at the center reduces the potential drop between the strips pairs which in turn increases the capacitance resulting in an increased current and a higher magnetic moment. This is also shown in comparing the electric field vectors in Fig. 2 (b) and Fig. 2 (d), where inserting the HRI materials results in more circulating arrows contributing to the magnetic dipole moment. In addition, Fig. 2 (c) shows that the HMIM has a more localized magnetic hot spot than the MIM. This is also consistent with the frequency red shift of the resonance as the capacitance increases. We also found the reflectance spectrum of the single HMIM on a glass substrate and the magnetic near field enhancement as a function of the HRI width (d) (see supplementary information).
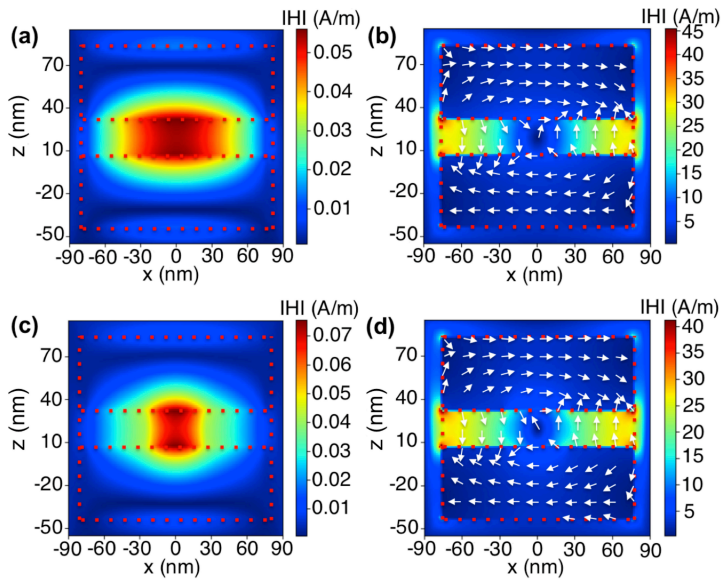

Fig. 2. (a) The magnetic near field $|H|$ at the magnetic resonance at the wavelength of $850.5 \mathrm{~nm}$ for a single MIM on a glass substrate without using the center HRI material (only the dielectric with 1.45 refractive index). (b) The near electric field $|E|$ at the same magnetic resonance in (a) and the corresponding electric field vectors. An enhancement factor $\left|H / H_{i}\right|^{2}=446.6$ is observed at the hot spot. (c) The magnetic near field $|H|$ at the magnetic resonance at the wavelength of $894.94 \mathrm{~nm}$ for a single HMIM on a glass substrate where the center HRI width (d) is $40 \mathrm{~nm}$. An enhancement factor $\left|H / H_{i}\right|^{2}=811.7$ is observed. (d) The near electric field $|E|$ at the same magnetic resonance in (c) and the corresponding electric field vectors.

The geometry of the MIM periodic array structure on a thin gold film on top of a glass substrate is shown in Fig. 3 (a). The thickness (t) of the gold film is $100 \mathrm{~nm}$, therefore the SPPs on the upper and lower surfaces of the film are decoupled. The glass substrate is of a thickness of $500 \mathrm{~nm}$ and a dielectric constant of 1.52. The HMIM dimensions is the same as that used for the single HMIM structure. A TM plane wave source illuminates the arrays from above at normal incidence along the $z$-direction with a wavelength ranging from 600 to $1000 \mathrm{~nm}$. We use perfectly matched layer boundary conditions (PML) above and below the arrays in the $z$-direction together with periodic boundary conditions along the $x$ and $y$ directions. The mesh accuracy is set to 3 which corresponds to a $\lambda / 14$ step size. A mesh override region of $1 \mathrm{~nm}$ is placed around the HMIM in the unit cell. For the magnetic near field enhancement plots, a 2D frequency-domain profile monitor in the $\mathrm{x}-\mathrm{Z}$ plane, is placed at the center of the structure in the unit cell, where its boundaries are the same as that of the structure. Fig. 3 (b) shows the reflection spectrum of the array at the position of maximum splitting when the period of the array is $837 \mathrm{~nm}$, where the higher and lower energy MP hybridized modes are at the wavelengths of $834.3 \mathrm{~nm}$ and $874.7 \mathrm{~nm}$ respectively.
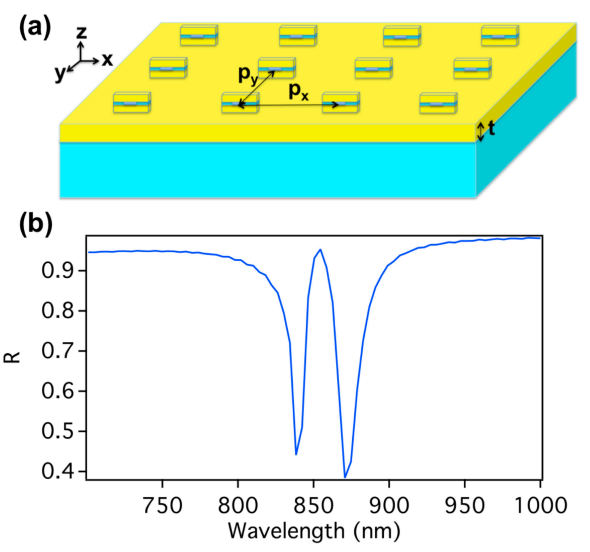

Fig. 3. (a) The geometry of the HMIM periodic array structure on a thin gold film on top of a glass substrate is shown in Fig. 3. The thickness ( $t$ ) of the gold film is $100 \mathrm{~nm}$. The glass substrate is of a thickness of $500 \mathrm{~nm}$ and a dielectric constant of 1.52 . The HMIM dimensions is the same as that used for the single HMIM structure. (b) the reflection spectrum of the array (the amount of power reflected normalized to the source power) at the position of maximum splitting when the period of the array is 837 $\mathrm{nm}$, where the higher and lower energy MP hybridized modes are at the wavelengths of $834.3 \mathrm{~nm}$ and $874.7 \mathrm{~nm}$ respectively.

Fig. 4 shows the positions of the reflection dips as a function of period. The horizontal black line is the magnetic resonance wavelength of the collective array of the HMIMs when placed in a periodic array on a gold film above a glass substrate (as in Fig. 3 (a) (without coupling to an SPP)), while the blue line indicates the resonance wavelengths of the $(1,0)$ SPP mode as a function of period. Both lines were obtained by simulations. The red lines represents the simulated reflection dips as the periods of the array $\left(\mathrm{p}_{\mathrm{x}}\right.$ and $p_{y}$ ) are both simultaneously changed from 625 to 925 in steps of $25 \mathrm{~nm}$. An anti-crossing behavior is observed 
generating two hybridized MP modes when the HMIM magnetic resonance (black line) and the SPP resonance (blue line) are approximately equal at a period of $837 \mathrm{~nm}$. Fig. 5 (a) and (b) shows the magnetic near field $|H|$ for the higher and lower energy MP hybridized modes at the wavelengths of $834.3 \mathrm{~nm}$ and $874.7 \mathrm{~nm}$ respectively corresponding to the position of maximum splitting at the period of $837 \mathrm{~nm}$. The magnetic near field enhancement factor is $\left|H_{H}\right|_{i}^{2}=1903$ and $\left|H / H_{i}\right|^{2}=3554.8$ at the higher and lower energy modes respectively, which is a further enhancement of $438 \%$ relative to the single HMIM and a total of $796 \%$ enhancement relative to a single MIM on a glass substrate structure. Note that the periodicity of the array itself away from the region of the coupling with the SPP, modifies the magnetic near field enhancement in agreement with far-field diffractive coupling results that has been previously studied $[10,15]$. For example, at the periods of $470 \mathrm{~nm}$ and $950 \mathrm{~nm}$, the maximum magnetic near field enhancement is $\left|H / H_{i}\right|^{2}=1858$ and $\left|H / H_{i}\right|^{2}=1787$ respectively. This gives a $229 \%$ and a $220 \%$ enhancement relative to the single HMIM on a glass substrate. In addition, compared to other structures such as high-index dielectric nanostructures [23, 24], the MIM structures provide higher localization and enhancement, however they are more complex in architecture and fabrication processes compared to highindex dielectric nanostructures, but the use of each depends on the specific application.

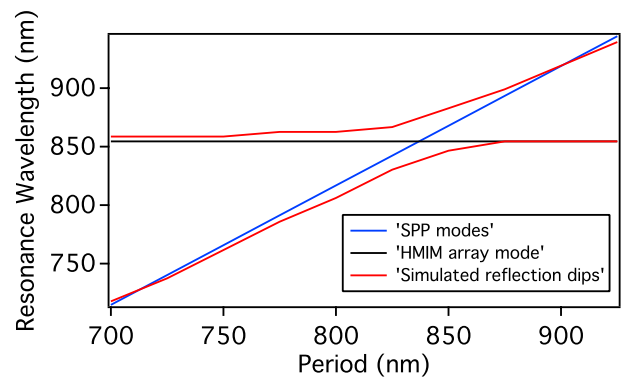

Fig. 4. The positions of the reflection dips as a function of period. The horizontal black line is the magnetic plasmon resonance of the collective array of the HMIMs when placed in a periodic array on a gold film above a glass substrate (as in Fig. 3 (without coupling to an SPP)), while the blue line indicates the resonance wavelengths of the $(1,0)$ SPP mode as a function of period. Both lines were obtained by simulations. The red lines represents the simulated reflection dips as the period of the array is changed from 620 to 925 in steps of $25 \mathrm{~nm}$.
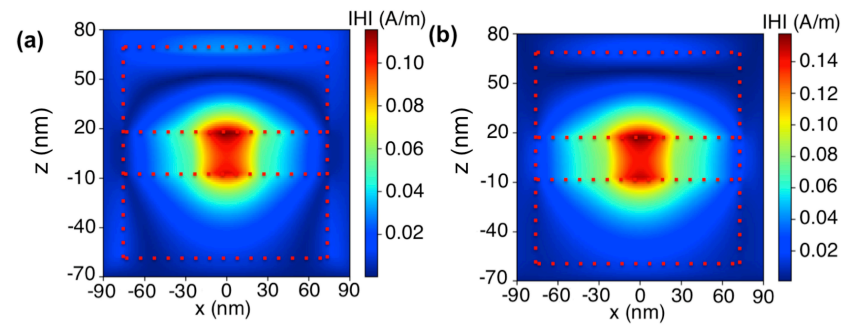

Fig. 5. The magnetic near field $|H|$ for the higher and lower energy MP hybridized modes at the wavelengths of $834.3 \mathrm{~nm}$ and $874.7 \mathrm{~nm}$ respectively corresponding to the position of maximum splitting at the period of $837 \mathrm{~nm}$.

\section{CONCLUSION}

In summary, we propose numerically an approach to narrow the plasmon linewidth and enhance the magnetic near field intensity at a magnetic hot spot in a hybridized metal-insulator-metal (HMIM) structure. The magnetic near field enhancement of the HMIM is increased by $82 \%$ compared with the MIM without the HRI material. We then couple this enhanced MP resonance to a propagating surface plasmon polariton (SPP) to achieve a further enhancement of $438 \%$, resulting in a total magnetic field enhancement at the gap of $\left|H / H_{i}\right|^{2}=3554.8$. Such highly enhanced and localized magnetic field has several potential applications including sensors, light matter interaction at weak magnetic fields and negative refractive index materials $[3,11]$.

\section{REFERENCES}

[1] L. D. Landau and E. M. Lifshitz, Electrodynamics of Continuous Media. (Wiley, 1984).

[2] A. K. Sarychev, Physical Review E 73, 036609 (2006).

[3] F. Monticone and Andrea Alu, J. Mater. Chem. 2, 9059-9072 (2014).

[4] M. Lorente-Crespo, L. Wang, R. Ortuño, C. García-Meca,Y. Ekinci and A. Martínez, Nano Lett. 13, 2654-2661 (2013)

[5] T. Pakizeh, M. S Abrishamian, N. Granpayeh, Opt. Express 14, 8240-8246 (2006).

[6] R.Verre, Z.J.Yang,T.Shegai,and M.Kall, Nano Lett. 15, 1952-1958 (2015).

[7] V. Shalaev, W. Cai, U. Chettiar, H. Yuan, A. Sarychev, V. Drachev, and A. Kildishev, Optics Lett. 30, 3356-3358 (2005).

[8] F. Shafiei, F. Monticone, K. Le, X. Liu, T. Hartsfield, A. Alu and X. Li, Nature Nanotechnology 8, 95-99 (2013).

[9] A. Vallecchi, M. Albani and F. Capolino, Optics Express 19, 2754-2772 (2011).

[10] C. J. Tang, P. Zhan, Z. S. Cao, J. Pan, Z. Chen, and Z. L. Wang, Physical Review B 83, 041402 (2011).

[11] V. Shalaev, Nature Photonics 1, 41 - 48 (2007).

[12] S. Maier, Plasmonics: fundamentals and applications (Springer, 2007).

[13] E. Hicks, S. Zou, G. Schatz, K. Spears and R. Van Duyne, Nano Lett. 5, 1065-1070 (2005).

[14] S. Zou, N. Janel and G. Schatz, J. Chem. Phys. 120, 10871 (2004).

[15] J. Chen, P. Mao, R. Xu, C. Tang, Y. Liu, Q. Wang and L. Zhang, Optics Express 23, 16238-16245 (2015).

[16] H. Liu, X. Sun, Y. Pei, F. Yao, and Y. Jiang, Opt. Lett. 36, 2414-2416 (2011).

[17] J. Chen, C. Tang, P. Mao, C. Peng, D. Gao, Y. Yu, Q. Wang and L. Zhang, IEEE Photonics Journal 8 (2016).

[18] J. Chen, T. Zhang, C. Tang, P. Mao, Y. Liu, Y. Yu, and Z. Liu, IEEE Photonics Technology Letters 28, 1529 - 1532 (2016).

[19] Z. Xi, Yonghua Lu, W. Yu, P. Yao, P. Wang and H. Ming, Optics Letters 38, 1591-1593 (2013).

[20] Yizhuo Chu and Kenneth B. Crozier, Opt. Lett. 34, 244-246 (2009).

[21] Johnson and Christy, "Optical Constants of Noble metals", Phys Rev B, 6, 4370 (1972).

[22] B. Ross and L. Lee, Optics Letters 34, 896-898 (2009).

[23] R. Bakker, D. Permyakov, Y. Feng Yu, D. Markovich, R. PaniaguaDomínguez, L. Gonzaga, A. Samusev, Y. Kivshar, B. Luk'yanchuk and A. Kuznetsov, Nano Letters 15, 2137-2142 (2015).

[24] P. Albella, M. Poyli, M. Schmidt, S. Maier, F. Moreno, J. JoséSaenz and J. Aizpurua, J. Phys. Chem. C 117, 13573-13584 (2015). 
